gु

\title{
Stress Transmission and Failure in Disordered Porous Media
}

\author{
Hadrien Laubie, ${ }^{1, *}$ Farhang Radjai, ${ }^{2,3, \dagger}$ Roland Pellenq, ${ }^{1,2,4,}$ and Franz-Josef Ulm ${ }^{1,2, \S}$ \\ ${ }^{1}$ Department of Civil and Environmental Engineering, Massachusetts Institute of Technology, \\ Cambridge, Massachusetts 02139, USA \\ ${ }^{2}\langle M S E\rangle^{2}$, UMI 3466 CNRS-MIT Energy Initiative, Massachusetts Institute of Technology, \\ 77 Massachusetts Avenue, Cambridge, Massachusetts 02139, USA \\ ${ }^{3}$ LMGC, CNRS-University of Montpellier, 163 rue Auguste Broussonnet, 34090 Montpellier, France \\ ${ }^{4}$ CINaM, CNRS-Aix Marseille Université, Campus de Luminy, 13288 Marseille Cedex 09, France
}

(Received 9 March 2017; published 14 August 2017)

\begin{abstract}
By means of extensive lattice-element simulations, we investigate stress transmission and its relation with failure properties in increasingly disordered porous systems. We observe a non-Gaussian broadening of stress probability density functions under tensile loading with increasing porosity and disorder, revealing a gradual transition from a state governed by single-pore stress concentration to a state controlled by multipore interactions and metric disorder. This effect is captured by the excess kurtosis of stress distributions and shown to be nicely correlated with the second moment of local porosity fluctuations, which appears thus as a (dis)order parameter for the system. By generating statistical ensembles of porous textures with varying porosity and disorder, we derive a general expression for the fracture stress as a decreasing function of porosity and disorder. Focusing on critical sites where the local stress is above the global fracture threshold, we also analyze the transition to failure in terms of a coarse-graining length. These findings provide a general framework which can also be more generally applied to multiphase and structural heterogeneous materials.
\end{abstract}

DOI: 10.1103/PhysRevLett.119.075501

Nearly all materials are porous on some scale either due to their basic structure as an assembly of elementary constituents (grains, fibers, etc.) or as a result of the insertion of pores in a host matrix during the manufacturing process [1]. As the pores tend to reduce the overall connectivity of the solid phase and stress-bearing capacity under load, bulk properties such as fracture strength and heat conduction crucially depend on the porous texture [2-23]. Amazingly, however, most upscaling models are essentially based on porosity as the only structural parameter and single-pore analysis [24], thus ignoring spatial pore distributions, pore interactions, and their effects on stress transmission.

Recent increasing interest in a better understanding of multipore behavior is motivated by the scope of tailoring porous texture as a means of controlling macroscopic properties. For example, reducing the stiffness of orthopedic implants by engineering the pores is important for their behavior in contact with bone [7,15-17,19,20]. In not only powder and sintered products such as pharmaceutical compacts [21] but also well-known homogeneous brittle materials such as glasses [25], the variability of mechanical properties may be reduced by taking advantage of the porous texture without lowering the porosity, which is sometimes required for functions such as impact resistance and adsorption. It is also important to mention here modern energy technologies that either require porous components as in batteries [26] or resort to fracking in order to extract organic material by interaction with the porous texture of the rock [27].
In this Letter, we consider a model porous system in which, besides porosity $\phi$, a parameter $I_{d}$ reflecting porosity fluctuations is introduced to characterize the porous texture. By varying $\phi$ and $I_{d}$ in a broad range of values, we generate ensembles of pore configurations in a linear elastic matrix subjected to tensile loading. As compared to a reference ordered structure $\left(I_{d}=0\right)$, the issue addressed here is how interpore stress distributions correlate with $I_{d}$ for varying $\phi$ and with fracture strength. Specifically, with regard to the classical single-pore stress analysis, an interesting question is whether a transition occurs from a state governed by local stress gradients to a state bearing global disorder.

Let us consider a square plate of side $L_{x}=L_{z}=L$ and thickness $L_{y} \ll L$. Porous textures can be generated by inserting circular pores (actually, cylinders of height $L_{y}$ ) of radius $R$ in the plate. We consider a reference ordered configuration defined by $N$ pores placed at the nodes of a square lattice such that $L=\sqrt{N} s$, where $s$ is the lattice step. The $2 \mathrm{D}$ porosity is given by $\phi=N \pi(R / L)^{2}$. Random configurations were generated from this reference system by applying Monte Carlo moves to the pores, with the requirement that the intercenter distance between two pores is above $2 \lambda R$ [28]. Metric randomness increases as $\lambda$ is decreased from $s /(2 R)$ to 1 . The number $N$ of pores is kept constant and porosity is controlled by changing $R$. To check the effectiveness of disorder for $\lambda=1$, we also used the grand canonical Monte Carlo method by randomly inserting 
pores of radius $R$ under the no-overlap condition. In this method, $R$ is kept fixed and porosity is controlled by the number of inserted pores. We also checked to see that the two-point probability function of the pore phase clearly reflects the evolution of disorder with $\lambda[28,29]$.

Various geometrical descriptors can be used to characterize random textures. Since the porosity $\phi$ is a mean global parameter, the next natural higher-order parameter is a measure of the local fluctuations of porosity. The definition of local porosity requires, however, a primitive volume that reflects pore distribution in the matrix. We use a square volume of length $\ell=\left(\pi R^{2} / \phi\right)^{1 / 2}$, which plays the same role as the mean free path in a gas and is reduced to the lattice step $s$ in the ordered reference system. The porosity $\phi_{i}$ at each point $i$ can thus be evaluated in a square volume $\ell^{2}$ centered on $i$. The disorder parameter $I_{d}$ is defined as the standard deviation of the set $\left\{\phi_{i}\right\}$ :

$$
I_{d}=\left[\left\langle\phi_{i}^{2}\right\rangle-\phi^{2}\right]^{1 / 2}
$$

which we refer to below as the "disorder index." For each value of $\phi$, this parameter can vary from zero for an ordered structure to a maximum value on the order of 0.18 for a fully disordered structure $(\lambda=1)$.

The matrix is a homogeneous brittle medium simulated by means of the lattice element method in which the volume is discretized as a lattice of elastic elements; see the Supplemental Material [30]. The material parameters were fixed to Young's modulus $E=1 \mathrm{MPa}$, Poisson's ratio $\nu=1 / 3$, and tensile strength $\sigma_{c}^{m}=\sigma_{c}(\phi \rightarrow 0)=3 \mathrm{kPa}$. The total strain at failure is $\sigma_{c}^{m} / E=0.3 \%$, implying that pore deformations are negligible and failure is essentially governed by stress concentration due to the porous texture. The bottom nodes along the $z$ direction were fixed and the porous samples were subjected to stepwise loading of the top nodes with zero boundary stress along the $x$ and $y$ directions until failure occurs at a fracture stress $\sigma_{c}$, depending on the porous texture. We performed a large number of simulations, with 25,121 , and 961 pores of increasing size up to a maximum porosity of $\phi=0.6$. The small numbers of pores were used to generate representative ensembles of configurations for different values of $\phi$ and $I_{d}$, repeating each simulation with ten independent pore configurations, whereas the large samples were used for a statistical analysis of stress fields. For these simulations, the spatial resolution of the matrix was always above five nodes per pore diameter.

Figure 1 displays four maps of vertical stresses $\sigma_{z z}$ normalized by the mean stress $\left\langle\sigma_{z z}\right\rangle$ for the same porosity $\phi=0.37$ but with increasing disorder $I_{d}$. Only tensile stresses (positive values) are shown since vertical stresses are mostly in tension. With the color scale being the same, we clearly observe stress chains with increasing inhomogeneity (shown up to 10 times the mean stress) as geometrical disorder increases. The probability density functions (PDFs) of stresses are shown in Fig. 2. They
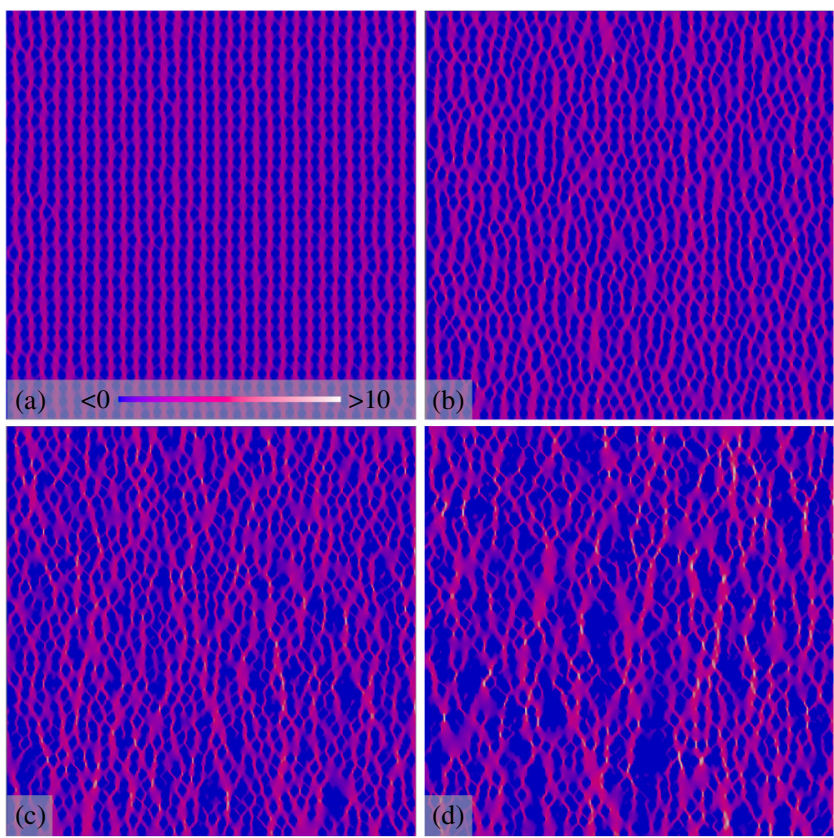

FIG. 1. Maps of the vertical tensile stress field in four different samples of porosity $\phi=0.37$ and increasing disorder: (a) $S_{1}$ with disorder index $I_{d}=0.008$, (b) $S_{2}$ with $I_{d}=0.087$, (c) $S_{3}$ with $I_{d}=0.120$, and (d) $S_{4}$ with $I_{d}=0.150$. The scale shows the range of normalized stresses varying from 0 and smaller values to 10 and larger values.

are characterized by a peak and nearly similar distribution around the peak centered on the mean stress for all PDFs but with a gradual broadening with increasing disorder. The PDF in the weakly disordered system $\left(S_{1}\right.$ with $\left.I_{d}=0.008\right)$ shows two modes and a nearly Gaussian falloff reflecting the close pore environment; see the Supplemental Material [30]. For stronger disorder, however, these features disappear and the PDFs become smooth with a nearly

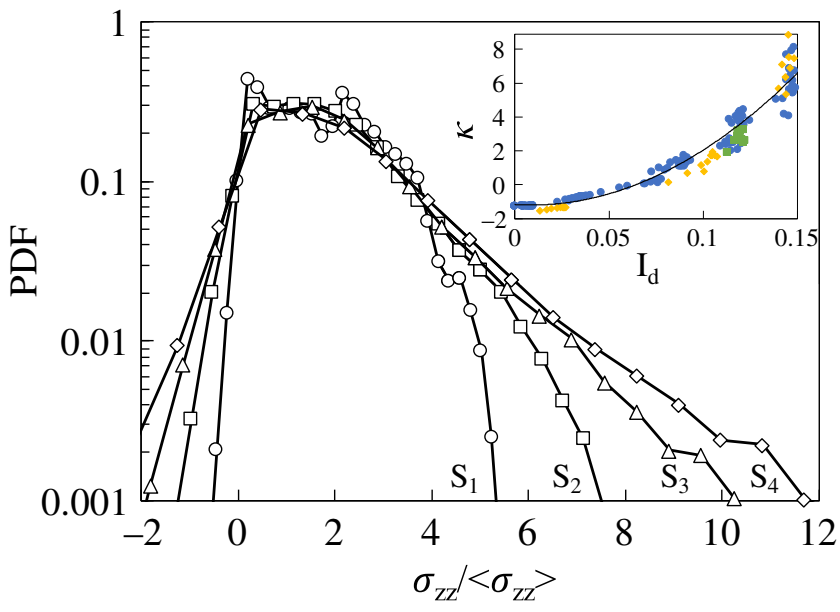

FIG. 2. Probability density functions (PDFs) of normalized vertical stresses for the four samples of Fig. 1. (Inset) The excess kurtosis $\kappa$ of tensile stresses as a function of disorder index $I_{d}$ for $\phi=0.2$ (green squares), $\phi=0.4$ (blue disks), and $\phi=0.5$ (yellow diamonds). The solid line is a quadratic fit. 
stretched exponential falloff in the range of positive (tensile) strong stresses as well as in negative (compressive) stresses. This behavior is quite generic, with a broadening of the PDF with increasing porosity; for more examples, see the Supplemental Material [30]. The transition from local pore environments to a disorder-induced stochastic distribution is interesting as it implies that fracture in this regime is governed not by single-pore environments but by disorder and its correlations.

The broadening of the PDFs is reflected in the excess kurtosis $\kappa=\left\langle\left(\sigma_{z z}-\left\langle\sigma_{z z}\right\rangle\right)^{4}\right\rangle /\left\langle\left(\sigma_{z z}-\left\langle\sigma_{z z}\right\rangle\right)^{2}\right\rangle^{2}-3$ of stresses, which is plotted in the inset to Fig. 2 as a function of the disorder index. We see that $\kappa$ increases quadratically with $I_{d}$, revealing thus a well-defined connection between porous texture and stress transmission. This relation is nearly independent of porosity. Note that $\kappa$ increases from -1 (which is the value for the Wigner semicircle distribution) to 0 (the value for a Gaussian) in $S_{1}$ and tending to 6 (for an exponential function) in $S_{4}$. The nearly exponential falloff of the stress PDFs at high disorder and porosity recalls the statistics of strong contact forces in a granular material [33-36]. This analogy can be elaborated by Delaunay triangulation of the matrix using the pore centers. In this representation, the matrix is tessellated into an assembly of triangular particles interacting with other triangles through contact lines joining neighboring pore centers. The mean stresses $T_{n}$ normal to all such lines define a coarse-grained ensemble. Examples of the stress maps are shown in the Supplemental Material together with their PDFs [30].

Since $I_{d}$ is well correlated with the excess kurtosis of stresses, which amplifies the contribution of extreme stress values, we expect the fracture strength $\sigma_{c}$ to be dependent on $I_{d}$ as well as on $\phi$. Figure 3(a) shows $\sigma_{c}$ normalized by tensile strength $\sigma_{c}^{m}$ of the matrix as a function $\phi$ for all of our simulation data. The upper bound represents the tensile strength $\sigma_{c}^{r}$ of our reference ordered configurations $\left(I_{d}=0\right)$. It can be evaluated by taking advantage of order and considering the effective cross section for stress transmission (see the Supplemental Material [30]):

$$
\sigma_{c}^{r}(\phi)=\sigma_{c}^{m}\left[1-(4 \phi / \pi)^{1 / 2}\right] .
$$

For each value of $\phi$, the tensile strength declines from this upper value as $I_{d}$ increases; see the direction of the arrow in Fig. 3(a). The extent of this strength weakening due to disorder is nearly the same for all values of $\phi$. For example, for $\phi>0.4$, the strength can vanish depending on the amount of disorder.

This behavior is obviously the effect of enhanced stress concentration observed in stress maps and PDFs with increasing disorder. The issue here is whether $I_{d}$ captures this disorder-induced weakening. Figure 3(b) displays the same data points but for $\sigma_{c}$ normalized by the reference strength $\sigma_{c}^{r}$ as a function of $I_{d}$. The average points are also shown for ten successive intervals of $I_{d}$ together with
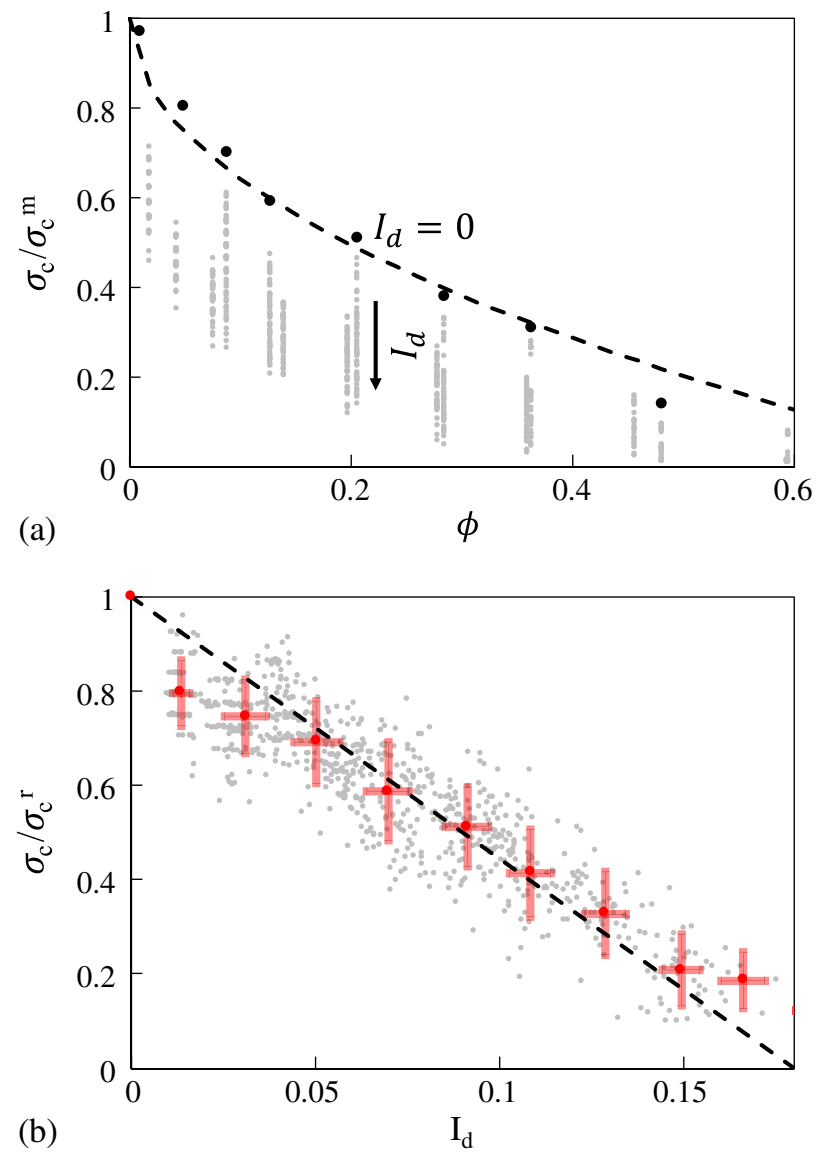

FIG. 3. (a) Tensile strength of all porous systems normalized by the matrix tensile strength $\sigma_{c}^{m}$ as a function of porosity $\phi$ for all values of disorder index $I_{d}$. The arrow shows the direction of increasing values of $I_{d}$. The dashed line is a fit by Eq. (2) and black markers correspond to ordered systems. (b) The same data normalized by the tensile strength $\sigma_{c}^{r}(\phi)$ of reference ordered systems as a function of the disorder index. The red symbols represent the averages and the standard variations of the data over ten intervals of $I_{d}$. The dashed line is a fit by Eq. (3).

their standard deviation. The data points are well fit by a linear function:

$$
\sigma_{c}\left(\phi, I_{d}\right) \simeq \sigma_{c}^{r}(\phi)\left(1-I_{d} / I_{d}^{\star}\right),
$$

with $I_{d}^{\star} \simeq 0.18$ representing a characteristic value of $I_{d}$ for which the strength vanishes and coincides with the maximum value of $I_{d}$. This value can occur only when $\phi>0.4$. For $\phi<0.4, I_{d}$ is always below $I_{d}^{\star}$. The effect of $\phi$ on fracture strength is fully included in that of ordered configurations $\sigma_{c}^{r}(\phi)$. We still observe a small variability around Eq. (3) that may be resolved by accounting for higher-order moments of local porosity fluctuations.

We now focus on the stress correlations and their relation with fracture. By definition, the material fails when the average stress $\sigma_{z z}$ over the whole volume equals $\sigma_{c}$. In the same way, under increasing load, a crack can propagate 
over a distance $d$ if the average stress $\sigma_{z z}(d)$ inside a 2D volume $d^{2}$ centered on the crack equals $\sigma_{c}$. Based on this picture, we proceed as follows. At a given level of the applied load $\sigma_{z z}$, we first determine all sites $i$ where $\sigma_{z z}^{i} \geq \sigma_{c}$. The number of these "critical sites" increases with loading. We then calculate the diameter $d_{i}$ of a circular probe centered on $i$ such that the average stress $\sigma_{z z}\left(d_{i}\right)$ inside the probe equals $\sigma_{c}$. The set $\left\{d_{i}\right\}$ of all probe diameters satisfying this condition thus provides a map of the stress state, as was also previously applied to the analysis of granular avalanches [40]. The average $\left\langle d_{i}\right\rangle$ is a "coarse-graining" length reflecting stress correlations, and the largest diameter $d_{\max }$ represents the most critical zone inside the system. In Figs. 4(a) and 4(b), two examples are shown with all critical sites and their sizes $d_{i}$ in color code.

Figure 4(c) displays the evolution of $d_{\max }$ as a function of loading for the four systems of Fig. 1. $d_{\max }$ increases exponentially as a function of the load, with values remaining small compared to system size, but at $\sigma_{z z} \simeq 0.75 \sigma_{c}$ it begins to diverge as nearly a power law $d_{\max }^{\alpha}$ towards system size, with exponent $\alpha$ on the order of 2 over a small range explored here. A finite-size analysis is necessary with more data points
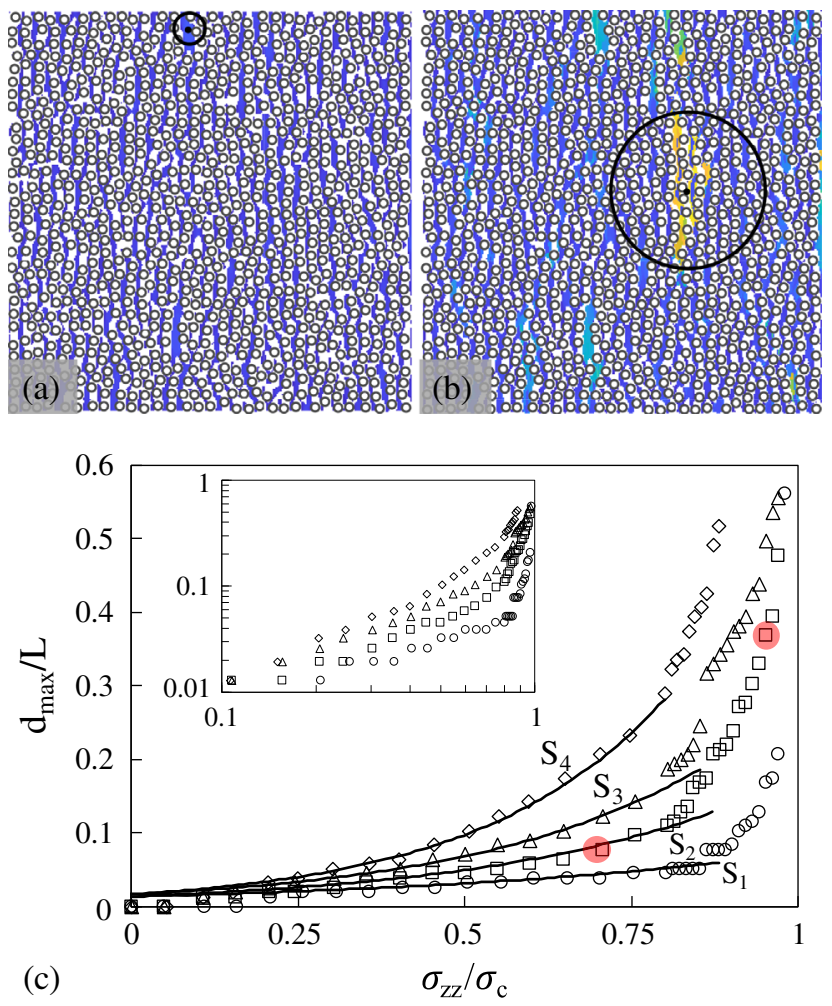

FIG. 4. (a),(b) Critical sites and their equivalent probe sizes encoded in color levels on a scale varying from blue (black) to yellow (light shade). The two black circles are the probes centered on the most critical sites at two instants of loading. (c) The diameter $d_{\max }$ of the most critical domain normalized by the system size $L$ as a function of vertical stress normalized by the system tensile strength for the four samples of Fig. 1. The solid lines are exponential fits. (Inset) The same data on a log-log scale. to obtain an accurate scaling behavior in this range. The mean length $\langle d\rangle$ behaves in a similar way but grows more slowly. $d_{\max }$ increases all the faster when the system is more disordered, which is consistent with the longer tails of the corresponding stress PDFs. The whole range of stresses between $\sigma_{z z} \simeq 0.75 \sigma_{c}$ and $\sigma_{z z}=\sigma_{c}$ corresponds to metastable states where global failure may occur as a result of fluctuations. Note also that, since pore deformations are negligibly small, the normalized stress PDFs do not evolve with loading. Only the mean stress increases proportionally to $\sigma_{z z}$, so the increase of $d_{\max }$ with loading is a consequence of the gradual sweeping of the stress PDF.

The nearly power-law divergence of $d_{\max }$ close to $\sigma_{c}$ means that the critical sites prevail and transition to failure may be described as a percolation process of clusters of sites. If a crack occurs inside such a cluster, it will propagate across the system. This picture is qualitatively similar to what is observed in avalanche initiation [40] and transition to failure in cohesive granular materials and porous rocks under compressive loading $[41,42]$ with a power-law divergence of cracking events. It should also be borne in mind that the value of $\sigma_{c}$ in the above analysis is a sample-to-sample fluctuating quantity. The metastable regime, as defined above, provides a rationale behind these fluctuations, with a distribution that may be described by the Weibull statistics [43].

The porous systems investigated in this Letter by means of extensive simulations highlight the role of multipore interactions and disorder in transition to failure. As porosity and/or disorder increase, this transition is increasingly controlled by stress distributions and correlations that we analyzed in terms of stress PDFs and a coarse-graining length. By quantifying the effect of metric disorder, our findings open a vast scope for the analysis of random porous materials with a broad range of porosities. Our results provide strong credit for the second order moment of local porosities as a (dis)order parameter that captures both the non-Gaussian broadening of stress distributions and the linear weakening of tensile strength with disorder, as described by Eq. (3). The elastic moduli undergo a similar weakening as a function of disorder [44]. Our approach and results cannot be directly applied to highly porous materials such as brittle foams, which are described in terms of bonds and topological disorder [2,45]. However, it is useful to extend this investigation to such extreme cases in order to assess the limits of the present analysis. Despite demanding computational resources, another important step beyond this work is the analysis of systems involving overlapped pores, broad pore size distributions, and open pores in 3D $[15,46]$. Finally, the methodology developed in this Letter can be applied to poroelastic behavior in disordered porous materials [24] and tested experimentally in materials of various degrees of disorder.

Research carried out within the X-Shale Hub@MIT, the Science and Engineering of Gas Shale, a collaboration 
between Shell, Schlumberger and MIT, enabled through MIT's Energy Initiative. F. R. would like to acknowledge the support of the ICoME2 Labex (ANR-11-LABX-0053) and the A MIDEX projects (ANR-11-IDEX-0001-02) cofunded by the French program "Investissements d'Avenir", managed by the French National Research Agency (Agence Nationale de la Recherche).

*hlaubie@mit.edu

†radjai@mit.edu

"pellenq@mit.edu

§ulm@mit.edu

[1] F. Dullien, Porous Media: Fluid Transport and Pore Structure (Academic Press, San Diego, 1992).

[2] K. Sieradzki and R. Li, Fracture Behavior of a Solid with Random Porosity, Phys. Rev. Lett. 56, 2509 (1986).

[3] F. Carvalho and J. Labuz, Experiments on effective elastic modulus of two-dimensional solids with cracks and holes, Int. J. Solids Struct. 33, 4119 (1996).

[4] N. Hu, B. Wang, G. W. Tan, Z. H. Yao, and W. F. Yuan, Effective elastic properties of 2-D solids with circular holes: numerical simulations, Compos. Sci. Technol. 60, 1811 (2000).

[5] J. Chung, Ph.D. thesis, University of Groningen, 2002.

[6] I.-h. Oh, N. Nomura, N. Masahashi, and S. Hanada, Mechanical properties of porous titanium compacts prepared by powder sintering, Scr. Mater. 49, 1197 (2003).

[7] C. Zou, E. Zhang, and M. Li, Preparation, microstructure and mechanical properties of porous titanium sintered by $\mathrm{Ti}$ fibers, J Mater Sci: Mater Med 19, 401 (2008).

[8] M. Cramer and I. Sevostianov, Effect of pore distribution on elastic stiffness and fracture toughness of porous materials, Int. J. Fract. 160, 189 (2009).

[9] P. M. Davis and L. Knopoff, The elastic modulus, percolation, and disaggregation of strongly interacting, intersecting antiplane cracks, Proc. Natl. Acad. Sci. U.S.A. 106, 12634 (2009).

[10] D. Hoey and D. Taylor, Quantitative analysis of the effect of porosity on the fatigue strength of bone cement, Acta Biomater. 5, 719 (2009).

[11] I. Sevostianov and V. Kushch, Effect of pore distribution on the statistics of peak stress and overall properties of porous material, Int. J. Solids Struct. 46, 4419 (2009).

[12] B. Li, B. Ã. Wang, and S. R. Reid, Effective elastic properties of randomly distributed void models for porous materials, Int. J. Mech. Sci. 52, 726 (2010).

[13] H. Yoshimura, A. Molisani, N. Narita, P. Cesar, and H. Goldenstein, Porosity dependence of elastic constants in aluminum nitride ceramics, Mater. Res. 10, 127 (2007).

[14] H. Shen, H. Li, and L. C. Brinson, Effect of microstructural configurations on the mechanical responses of porous titanium: A numerical design of experiment analysis for orthopedic applications, Mech. Mater. 40, 708 (2008).

[15] B. Q. Li, F. Yan, and X. Lu, Effect of microstructure on the tensile property of porous $\mathrm{Ti}$ produced by powder metallurgy technique, Mater. Sci. Eng. A 534, 43 (2012).
[16] J. Choren, S. Heinrich, and M. Silver-Thorn, Young's modulus and volume porosity relationships for additive manufacturing applications, J. Mater. Sci. 48, 5103 (2013).

[17] B. Q. Li, C. Y. Wang, and X. Lu, Effect of pore structure on the compressive property of porous Ti produced by powder metallurgy technique, Mater. Des. 50, 613 (2013).

[18] A. V. Manoylov, F. M. Borodich, and H. P. Evans, Modelling of elastic properties of sintered porous materials, Proc. R. Soc. A 469, 20120689 (2013).

[19] L. Reig, C. Tojal, D. J. Busquets, and V. Amigó, Microstructure and mechanical behavior of porous Ti-6Al-4V processed by spherical powder sintering, Materials 6, 4868 (2013).

[20] H. Attar, M. Calin, L. C. Zhang, K. Zhuravleva, A. Funk, S. Scudino, C. Yang, and J. Eckert, Comparative study of microstructures and mechanical properties of in situ Ti-TiB composites produced by selective laser melting, powder metallurgy, and casting technologies, J. Mater. Res. 29, 1941 (2014).

[21] Ö. Keles, N. P. Barcenas, D. H. Sprys, and K. J. Bowman, Effect of porosity on strength distribution of microcrystalline cellulose, AAPS PharmSciTech 16, 1455 (2015).

[22] J. Jeong and H. Ram, Porous media modeling and microstructurally motivated material moduli determination via the micro-dilatation theory, Eur. Phys. J. Spec. Top. 224, 1805 (2015).

[23] C. Wang and B. Li, Finite element simulation of pore morphology and stress distribution of porous titanium, Adv. Mater. Res. 1120-1121, 932 (2015).

[24] L. Dormieux, D. Kondo, and F.-J. Ulm, Microporomechanics (John Wiley \& Sons, New York, 2006).

[25] J. Vasseur, F. Wadsworth, Y. Lavallée, A. Bell, I. Main, and D. Dingwell, Heterogeneity: the key to failure forecasting, Sci. Rep. 5, 13259 (2015).

[26] H. Lee, M. Yanilmaz, O. Toprakci, K. Fu, and X. Zhang, A review and recent developments in membrane separators for rechargeable lithium-ion batteries energy \& environmental science, Energy Environ. Sci. 7, 3857 (2014).

[27] Q. Li, H. Xing, J. Liu, and X. Liu, A review on hydraulic fracturing of unconventional reservoir, Petroleum 1, 8 (2015).

[28] P. Smith and S. Torquato, Computer simulation results for the two-point probability function of composite media, J. Comput. Phys. 76, 176 (1988).

[29] S. Torquato, Random Heterogeneous Materials. Microstructure and Macroscopic Properties (Springer-Verlag, New York, 2002).

[30] See Supplemental Material at http://link.aps.org/ supplemental/10.1103/PhysRevLett.119.075501, which includes Refs. [31-39], for brief descriptions of the lattice element method and of the generation of disorder, the stress statistics at different porosities and around a single pore, the tensile strength of ordered configurations and a coarsegraining analysis.

[31] H. Laubie, F. Radjaï, R. Pellenq, and F.-J. Ulm, A potentialof-mean-force approach for fracture mechanics of heterogeneous materials using the lattice element method, J. Mech. Phys. Solids 105, 116 (2017).

[32] E. G. Kirsch, Die theorie der elastizität und die bedürfnisse der festigkeitslehre, Z. Ver. Dtsch. Ing. 42, 797 (1898).

[33] F. Radjai, M. Jean, J.-J. Moreau, and S. Roux, Force distributions in dense two-dimensional granular systems, Phys. Rev. Lett. 77, 274 (1996). 
[34] V. Topin, F. Radjai, and J.-Y. Delenne, Subparticle stress fields in granular solids, Phys. Rev. E 79, 051302 (2009).

[35] C. Voivret, F. Radjai, J.-Y. Delenne, and M. S. El Youssoufi, Multiscale force networks in highly polydisperse granular media, Phys. Rev. Lett. 102, 178001 (2009).

[36] F. Radjai, Modeling force transmission in granular materials, C. R. Phys. 16, 3 (2015).

[37] H. Herrmann and S. Roux, Statistical Models for the Fracture of Disordered Media, Random Materials and Processes (Elsevier Science, New York, 1990).

[38] V. Topin, J.-Y. Delenne, F. Radjaï, L. Brendel, and F. Mabille, Strength and failure of cemented granular matter, Eur. Phys. J. E 23, 413 (2007).

[39] H. Laubie, S. Monfared, F. Radjaï, R. Pellenq, and F.-J. Ulm, Effective potentials and elastic properties in the lattice element method: isotropy and transverse isotropy, J. Nanomech. Micromech. 7, 4017007 (2017).

[40] L. Staron, J.-P. Vilotte, and F. Radjai, Preavalanche instabilities in a granular pile, Phys. Rev. Lett. 89, 204302 (2002).
[41] F. Kun, I. Varga, S. Lennartz-sassinek, and I. G. Main, Approach to failure in porous granular materials under compression, Phys. Rev. E 88, 062207 (2013).

[42] A. F. Bell, M. Naylor, and I. G. Main, The limits of predictability of volcanic eruptions from accelerating rates of earthquakes, Geophys. J. Int. 194, 1541 (2013).

[43] D.-H. Nguyen, E. Azéma, P. Sornay, and F. Radjai, Bondedcell model for particle fracture, Phys. Rev. E 91, 022203 (2015).

[44] H. Laubie, S. Monfared, F. Radjaï, R. Pellenq, and F.-J. Ulm, Disorder-induced stiffness degradation of highly disordered porous materials, J. Mech. Phys. Solids, DOI:10.1016/j.jmps.2017.05.008 (2017).

[45] P. M. Duxbury, S. G. Kim, and P. L. Leath, Size effect and statistics of fracture in random materials, Mater. Sci. Eng. A 176, 25 (1994).

[46] K. Ioannidou, K. J. Krakowiak, M. Bauchy, C. G. Hoover, E. Masoero, S. Yip, F.-J. Ulm, P. Levitz, R. J.-M. Pellenq, and E. Del Gado, Mesoscale texture of cement hydrates, Proc. Natl. Acad. Sci. U.S.A. 113, 2029 (2016). 